\title{
Integration of Extreme-point Symmetric Mode Decomposition and Singular Value Decomposition for Dynamic Deflection Denoising of Urban Bridges Obtained by Ground-based Microwave Interferometry
}

\author{
Mengzhuo Jiang, Xianglei Liu, ${ }^{*}$ Hui Wang, and Yimeng Huang ${ }^{* *}$ \\ Key Laboratory for Urban Geomatics of National Administration of Surveying, Mapping and Geoinformation, \\ Beijing University of Civil Engineering and Architecture, 1 Zhanlanguan Road, Beijing 100044, P. R. China
}

(Received September 29, 2020; accepted November 23, 2020)

Keywords: bridge signal, ground-based microwave interferometry, signal denoising, singular value decomposition, extreme-point symmetric mode decomposition

Ground-based microwave interferometry has been used extensively for dynamic deflection measurements of bridges. The Imaging by Interferometric Survey of Structures (IBIS-S) instrument is a system based on ground-based microwave interferometry, which consists of radar sensors for transmitting and receiving microwave and corresponding auxiliary units. To suppress the noise of the dynamic deflection of urban bridges obtained by ground-based microwave interferometry, by integrating singular value decomposition (SVD) and extremepoint symmetric mode decomposition (ESMD), a novel ESMD-SVD denoising method is proposed in this paper. First, the original bridge signal obtained by the IBIS-S sensor was decomposed to obtain a series of intrinsic mode functions (IMFs) and a signal trend term by ESMD. Second, the signal trend term was extracted and the IMFs were reconstructed, which removed the effect of the signal trend term on the singular value selection in SVD. SVD was used to eliminate the random noises and other noises in the reconstructed signal. Finally, the denoised signal obtained by SVD was superimposed on the trend term to obtain the final denoised signal. The results of both simulated and on-site experiments showed that the proposed ESMD-SVD method had a powerful signal denoising ability.

\section{Introduction}

Ground-based microwave interferometry is a new technology with the advantages of realtime monitoring, noncontact measurement, high distance resolution, high precision, and allweather and all-day measurements. ${ }^{(1-3)}$ The Imaging by Interferometric Survey of Structures (IBIS-S) instrument is a system based on ground-based microwave interferometry, which consists of radar sensors for transmitting and receiving microwave and corresponding auxiliary units. ${ }^{(1-3)}$ It has been used extensively for dynamic deflection measurements of bridges in recent years. ${ }^{(4-6)}$ Most urban bridges are built in complex environments, such as rivers or other

\footnotetext{
*Corresponding author: e-mail: liuxianglei@bucea.edu.cn

** Corresponding author: e-mail: 15011329653@163.com

https://doi.org/10.18494/SAM.2020.3126
} 
places with steep terrain with high pedestrian and vehicle use. In the process of obtaining bridge dynamic deflection using the IBIS-S sensor, various factors, such as the surrounding environment, ground motion, complex traffic activities, human operation, and the equipment itself, will inevitably produce noise in the collected dynamic deflection of urban bridges, which can lead to data analysis errors..$^{(7,8)}$ Therefore, signal noise reduction has always been a very important step in signal processing.

At present, primary signal denoising methods include the wavelet transform (WT), empirical mode decomposition (EMD), ensemble empirical mode decomposition (EEMD), and singular value decomposition (SVD). WT has good local properties in the time-frequency domain, can effectively process random and nonstationary signals, and has been widely used in denoising nonstationary vibration signals. ${ }^{(9-12)}$ However, the denoising effect of WT is greatly affected by the wavelet basis and the decomposition level and threshold. ${ }^{(9-12)}$ In WT noise reduction, it is necessary to manually select the appropriate wavelet base, the number of wavelet decomposition layers, and the noise reduction threshold, which cause a lack of adaptability. ${ }^{(13,14)}$ In addition, WT is not suitable for nonlinear signals, which means that it is not suitable for analyzing the dynamic deflection signals of monitored urban bridges. If a monitored bridge is damaged, the obtained dynamic deflection should be a typical nonlinear and nonstationary signal. ${ }^{(14,15)}$ EMD is an adaptive decomposition method proposed by Huang et al. in 1998, which overcomes the limitations of wavelet analysis. ${ }^{(16)}$ It can adaptively decompose a signal into a series of intrinsic mode functions (IMFs) of different scales without artificially selecting the basis function and can achieve the adaptive filtering of the signal. It has been widely used in the field of vibration signal denoising. ${ }^{(14,15)}$ However, EMD can cause the mode mixing problem, which means that signals of different frequencies or scales appear in the same IMF component, or signals of the same scale or frequency are automatically decomposed into multiple different IMF components. ${ }^{(15,17)}$ Moreover, the effect of this recursive mode is affected by the envelope mode and there is a problem of a rough trend function. ${ }^{(15,17)}$ EEMD is a method integrating the statistical properties of a Gaussian white noise signal and EMD. In EEMD, Gaussian white noise is added to the signal, then EMD is performed to obtain more reliable IMFs, ${ }^{(15,18-24)}$ which maintains the advantages of the EMD method while reducing the mode mixing problem. This method has been widely used in signal processing. ${ }^{(18-24)}$ However, if the amplitude of the added noise and the number of collective trials are not appropriate, more IMF components will be produced and there will be a greater error in the results. ${ }^{(15-24)}$ Nonlinear and nonstationary signals measured with a low signal-to-noise ratio (SNR) often have strong random and impulse noises, and the useful signals are almost submerged by noise. ${ }^{(24-29)}$ SVD is an effective signal processing technique for analyzing nonlinear and nonstationary signals, which has been widely used in speech recognition, image processing, fault diagnosis, signal denoising, and various other fields. ${ }^{(24-29)}$ Because signal information and noise information have different effects on the singular values of the matrix, SVD extracts signal features and separates noise by selecting appropriate singular values of the matrix. ${ }^{(24-29)}$ The phase shift of the denoised signal obtained by SVD is small and there is no time delay. ${ }^{(24-29)}$ However, the denoised results of SVD will be affected by the signal trend term. ${ }^{(29)}$ Extreme-point symmetric mode decomposition (ESMD) is a new analysis method in the time-frequency domain. It is an improved method based on 
EMD and EEMD, and is being increasingly applied in fields including mechanical vibration, marine and atmospheric sciences, and seismology. ${ }^{(1,4,14,15,30)}$ ESMD uses direct interpolation (DI) for spectrum analysis, which can not only intuitively reflect the time-varying amplitude and frequency of each mode, but also clarify the instantaneous energy change. ${ }^{(1,14,15)}$ Moreover, this method uses the least-squares method to optimize the final residual mode to obtain the best adaptive global mean (AGM) curve..$^{(1,14,15)}$ This new method can effectively reduce the difficulty in determining the number of modal decomposition screenings and the effect of modal aliasing. Although ESMD is superior to EMD and EEMD in all aspects, it still has problems, for example, noises with different frequencies and scales are in the decomposed IMFs obtained by ESMD. ${ }^{(15)}$

It is difficult to effectively extract useful signal details from strong noise using only one of the above methods for denoising signals. Yang et al. proposed a WT-SVD bilayer filtering denoising method based on WT and SVD denoising processes, and experimentally proved its effectiveness. ${ }^{(28)}$ However, this method did not reduce the effect of the signal trend term on SVD. To solve the mode mixing problem in EMD, Wen and Zhou proposed an SVD-EEMD method combining SVD and EEMD that can extract the fault information of a rolling bearing effectively and realize a precise fault diagnosis. ${ }^{(29)}$ However, the added Gaussian white noise will influence the effect of noise reduction.

With this background, the ESMD-SVD method is proposed for the dynamic deflection signal denoising of urban bridges obtained by ground-based microwave interferometry. ESMD is used to decompose the original obtained bridge signal into a series of IMFs and a signal trend term. SVD is used to eliminate the random noises and other noises in the reconstructed signal. Then, the signal obtained by SVD is superimposed on the trend term to obtain the final denoised signal, so as to realize the complementary advantages of SVD and ESMD. Finally, the signal after the noise reduction by SVD is superimposed on the trend item to obtain the final denoised signal.

\section{Methods}

Figure 1 shows the entire proposed ESMD-SVD denoising method. The original obtained bridge signal is decomposed into a series of IMFs and a signal trend term R using ESMD. Then, the signal trend term is extracted and the IMFs are reconstructed into a new signal. SVD is used to remove noises from the reconstructed signal. The denoised signal obtained by SVD and the trend term are reconstructed into the final denoised signal.

\subsection{Signal trend extraction}

ESMD was used to decompose the original bridge signal into a series of IMFs and an AGM curve. The AGM curve was considered as the best trend term of the original bridge signal. Then, the signal trend term was extracted and the IMFs were reconstructed, which overcame the effect of the signal trend term on the singular value selection in SVD. For the original bridge signal $y(t)$, the process of signal trend extraction is as follows. 


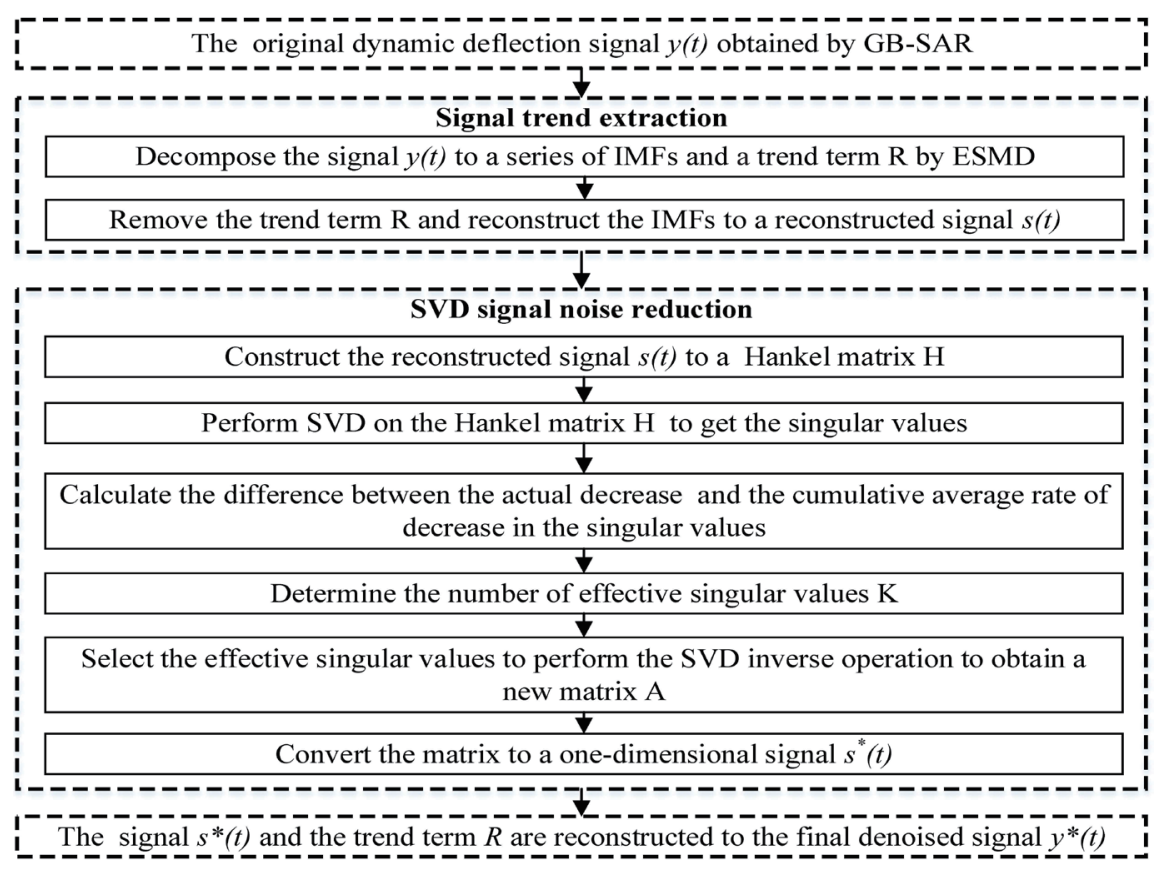

Fig. 1. Workflow of ESMD-SVD denoising method.

Step 1: Find all the maximum and minimum extremal points of the original bridge signal $y(t)$ and define the midpoints of two adjoining extremal points as $F_{i}(i=1,2, \ldots, n-1)$. Use linear interpolation to obtain the midpoints of the left and right boundaries as $F_{0}$ and $F_{n}$, respectively.

Step 2: Use cubic spline interpolation to obtain curves as $L_{i}(i=1,2, \ldots, m, m>1)$ based on $N+1$ points in $F_{i}(i=1,2, \ldots, n)$. Then, calculate the average of these curves and define the average curve as $\widetilde{L}=\left(L_{1}+L_{2}+\ldots+L_{m}\right) / m$.

Step 3: Calculate $y(t)-\widetilde{L}$ by repeating Step 1 to obtain $I M F_{1}$ until $|\widetilde{L}| \leq \varepsilon$ is satisfied or the value of the sifting time term reaches the preset maximum value $K$. In this study, the maximum allowable error $\varepsilon$ was set to $0.001 \sigma_{0}$, where $\sigma_{0}$ is the standard deviation of the original data $y(t)$ and the total average of the original data $y(t)$.

Step 4: Repeat Steps 1 to 3 for $y(t)-I M F_{i}$ to obtain the remainder $I M F_{1}, \ldots, I M F_{n}$ and a residual $r$ that does not exceed a certain number of extreme points. Then, calculate the variance $\sigma$ of $y(t)-r$.

Step 5: Change the value of the sifting time term $K$ in a finite integer interval on $\left[K_{\min }, K_{\max }\right]$. Then, repeat Steps 1 to 4 and obtain the optimal sifting time term $K_{0}$ with the minimum variance.

Step 6: Under the conditions of the optimal sifting time value $K_{0}$ and the optimal allowable error $\varepsilon$, the original bridge signal $y(t)$ is optimally decomposed to obtain a series of IMFs and an optimal AGM curve. The AGM curve is considered as the best trend term $R$ of the original bridge signal $y(t)$.

Step 7: Remove the trend term $R$ and reconstruct the IMFs obtained by the decomposition in Step 6 into a reconstructed signal $S(t)$. 


\subsection{Signal noise reduction in SVD}

The principle of signal noise reduction in SVD is as follows. ${ }^{(1)}$ Construct the signal containing noise into an $m \times n$-order matrix $\boldsymbol{H}^{(2)}$ Perform SVD on the matrix $\boldsymbol{H}$ to obtain a singular value matrix $\boldsymbol{Q} . \boldsymbol{Q}$ is composed of the singular value $\sigma_{i}$ of the signal. ${ }^{(3)}$ Set the corresponding singular value of the noise information to zero, and then use the SVD inverse operation to reconstruct a new signal to obtain the denoised signal. SVD employs two key technologies in signal denoising: the selection of the construction matrix and the determination of the effective singular value. Generally constructed matrix forms are the Toeplitz, cycle, and Hankel matricies. ${ }^{(27-29)}$ There are many methods to determine the order of singular values, such as the entropy increment, singular value difference spectrum, and singular value variance methods. ${ }^{(27-29)}$ In this paper, the Hankel matrix is used to construct a one-dimensional signal into a matrix, and the singular value accumulation method proposed by Meng et al. is used to determine the number of effective singular values. ${ }^{(27)}$ This method calculates the difference between the actual decrease in the number of singular values and the cumulative average rate of decrease in the number of singular values, and uses the point corresponding to the maximum difference as the boundary point of the effective singular values to determine the number of effective singular values. ${ }^{(27)}$ For the reconstructed signal $S(t)$ obtained in Step 7 above, the detailed process of signal noise reduction by SVD is as follows.

Step 8: For the reconstructed signal $S(t)$, construct a matrix in the form of Eq. (1) to form an $m \times n$-order Hankel matrix $\boldsymbol{H}$,

$$
\boldsymbol{H}_{m \times n}=\left[\begin{array}{cccc}
s(1) & s(2) & \cdots & s(n) \\
s(2) & s(3) & \cdots & s(n+1) \\
\vdots & \vdots & & \vdots \\
s(m) & s(m) & \cdots & s(N)
\end{array}\right]
$$

where $N$ is the length of the reconstructed signal $S(t), 1<m<N$ and $1<n<N$. According to Eqs. (2) and (3), choose the row number $m$ and column number $n$ to make the Hankel matrix $\boldsymbol{H}$ closer to a square matrix.

$$
\begin{gathered}
m=\left\{\begin{array}{l}
(N+1) / 2, \text { if } N \text { is odd } \\
N / 2, \quad \text { if } N \text { is even }
\end{array}\right. \\
n=N+1-m
\end{gathered}
$$

Step 9: Perform SVD on the Hankel matrix $\boldsymbol{H}$ to obtain the following decomposition form:

$$
\boldsymbol{H}_{m \times n}=\boldsymbol{U}_{m \times m} \boldsymbol{Q}_{m \times n} \boldsymbol{V}_{n \times n}^{T} .
$$

Here, $\boldsymbol{U}$ is an $m \times n$-order left orthogonal matrix, $\boldsymbol{V}$ is an $n \times n$-order right orthogonal matrix, and $\boldsymbol{Q}$ is an $m \times n$ diagonal matrix. $\boldsymbol{Q}$ can also be expressed as 


$$
\boldsymbol{Q}=\left[\begin{array}{llll}
\sigma_{1} & & & 0 \\
& \sigma_{2} & & \\
& & \ddots & \\
0 & & & \sigma_{q}
\end{array}\right],
$$

where $\sigma_{1}>\sigma_{2}>\ldots>\sigma_{q}>0$ and $\sigma_{i}(i=1,2, \ldots, q)$ are the singular values of the Hankel matrix $\boldsymbol{H}$. $q$ is the total number of singular values obtained by decomposition.

Step 10: Using the singular value accumulation method proposed by Meng et al., calculate the difference between the actual decrease in the number of singular values and the cumulative average rate of decrease in the number of singular values using Eqs. (6)-(8).

$$
\begin{gathered}
d_{i}=\frac{\sigma_{1}-\sigma_{i}}{i-1} \times(i-1)=\sigma_{1}-\sigma_{i} \\
d_{0}=\frac{\sigma_{1}-\sigma_{q}}{q-1} \times(i-1) \\
D_{i}=d_{i}-d_{0}
\end{gathered}
$$

Here, $d_{i}$ is the cumulative number of singular values, $d_{0}$ is the cumulative average rate of decrease in the number of singular values, and $D_{i}$ is the singular value cumulative difference. Also, $1<i \leq q$, where $q$ is the total number of singular values obtained by decomposition. $\sigma_{i}$ is the $i$ th singular value.

Step 11: According to Eq. (9), when $D_{i}$ takes its maximum, the number of effective singular values, $k$, is equal to $i$.

$$
\text { if } D_{i}=\max D_{i}, \quad k=i
$$

Because signal information and noise information have different effects on the singular values of the Hankel matrix $\boldsymbol{H}$, the effective signal is mainly reflected by the $k$ larger singular values in the former part, while the noise signal is reflected by the smaller singular values in the latter part. When $i<k$, more effective singular values are included with increasing $i$ and the rate of decrease in the number of effective singular values is generally high, so $D_{i}$ will increase with $i{ }^{(26)}$ In contrast, when $i>k$, more singular values of noise are included with increasing $i$ and the rate of decrease in the number of noise singular values is usually low, so $D_{i}$ will decrease with increasing $i .^{(26)}$ Therefore, the maximum $D_{i}$ will appear at the intersection of the effective singular values of the signal and the singular values of the noise.

Step 12: Retain the first $k$ effective singular values of the main diagonal matrix $\boldsymbol{S}$ and then set all other small singular values to 0 to obtain a new main diagonal matrix $\boldsymbol{S}^{\prime}=$ $\operatorname{diag}\left(\sigma_{1}, \sigma_{2}, \ldots, \sigma_{k}, 0, \ldots, 0\right)$, where $k<q$ and $\sigma_{i} \neq 0, i=1,2, \ldots, k$. 
Step 13: The new diagonal matrix $\boldsymbol{Q}^{\prime}$, the left orthogonal matrix $\boldsymbol{U}$, and the right orthogonal matrix $\boldsymbol{V}$ are substituted into Eq. (4) to obtain a new matrix $\boldsymbol{A}$.

Step 14: Convert the matrix $\boldsymbol{A}$ to a one-dimensional signal $S^{*}(t)$.

Step 15: The final denoised signal $y^{*}(t)$ is obtained by the superposition of the signal $S^{*}(t)$ and the trend term $\mathrm{R}$.

\section{Simulated Experiments and Analysis}

In order to verify the feasibility and accuracy of the proposed ESMD-SVD denoising method, simulated experiments were carried out. The original bridge signal obtained by ground-based microwave interferometry is a complex nonstationary signal contaminated by some noise. Therefore, a nonlinear and nonstationary analog signal $Y(t)$, which consists of three basic analog signals with different frequencies and a manually added Gaussian white noise signal with $20 \mathrm{~dB}$ SNR, was generated. The expressions of the three basic analog signals and the analog signal $Y(t)$ are as follows.

$$
\begin{gathered}
X_{1}(t)=\sin (6 \pi t) \\
X_{2}(t)=\cos (5 \pi t) \\
X_{3}(t)=\cos (20 \pi t+2 \sin (3 \pi t)) \\
Y(t)=X_{1}(t)+X_{2}(t)+X_{3}(t)+w(t)=X(t)+w(t)
\end{gathered}
$$

Here, $w(t)$ is a Gaussian white noise signal and the analog signal $X(t)$ is composed of three basic analog signals without noise. The waveforms of the analog signal, $Y(t)$ and $X(t)$, and the three basic analog signals are shown in Fig. 2.

The analog signal $Y(t)$ was first decomposed into nine IMFs and one signal trend term $\mathrm{R}$ by ESMD. The nine IMFs have corresponding significant natural frequencies, which are arranged from high frequency to low frequency, as shown in Fig. 3. Then, the nine IMFs obtained by ESMD are added to obtain the reconstructed signal $S(t)$.

The sampling time of the analog signal $Y(t)$ is $3 \mathrm{~s}$ and the number of sampling points is 3001. Thus, the length $N$ of the reconstructed signal $S(t)$ is 3001 and $N$ is an odd number. For the reconstructed signal $S(t)$, a $1501 \times 1501$-order Hankel matrix $\boldsymbol{H}$ was constructed using Eqs. (1)-(3). The SVD of the Hankel matrix $\boldsymbol{H}$ was performed, and 1501 singular values were obtained. Since the number of singular values is large and the final singular values are small, only the first 200 singular values were selected to form the SVD curve shown in Fig. 4(a). Using Eqs. (6)-(8), all singular values were used to calculate the cumulative differences of the singular values. The singular value cumulative difference distribution curve was composed of the cumulative differences of the first 200 singular values as shown in Fig. 4(b). 


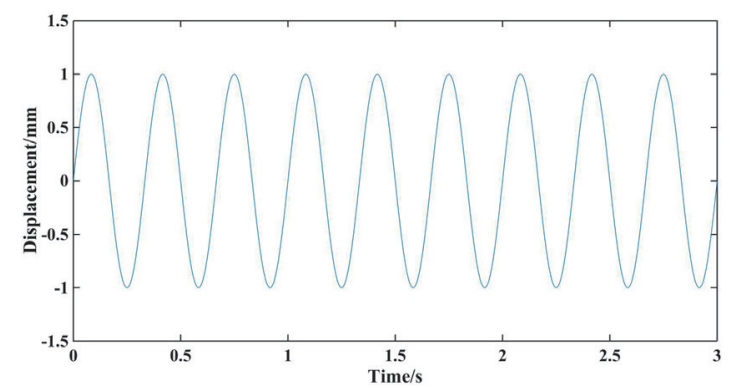

(a)

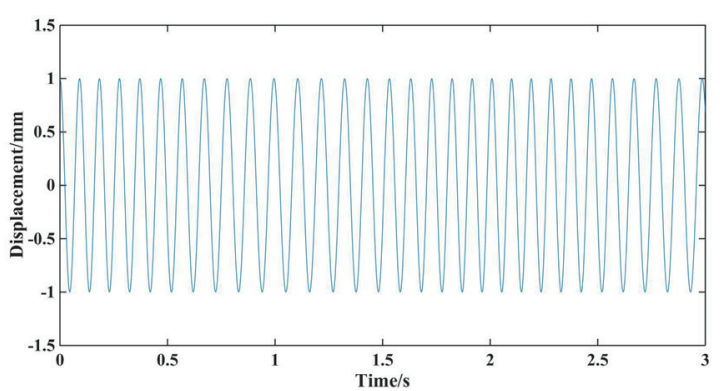

(c)

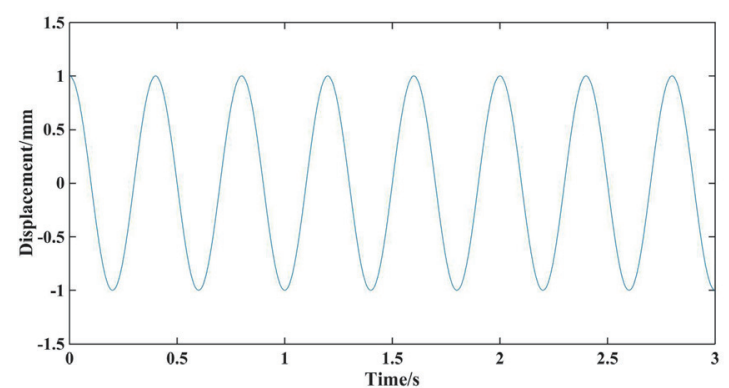

(b)

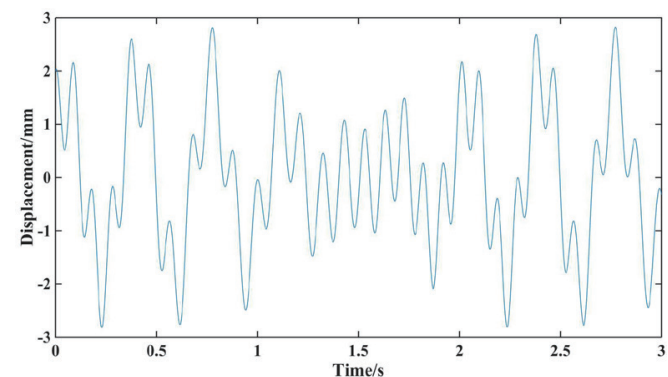

(d)

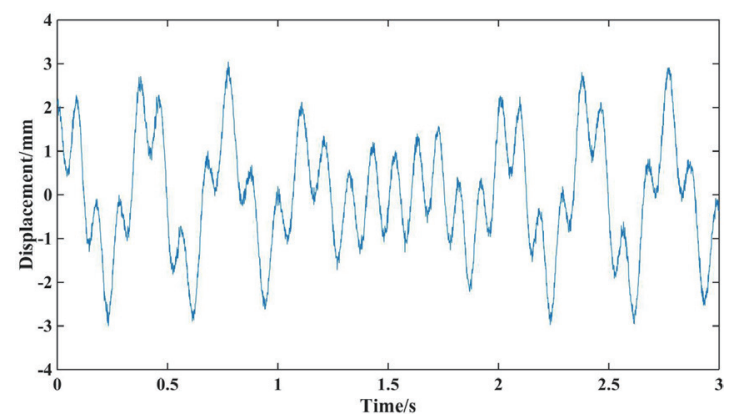

(e)

Fig. 2. (Color online) Waveforms of analog signal: (a) waveform of basic analog signal $X_{1}(t)$, (b) waveform of basic analog signal $X_{2}(t)$, (c) waveform of basic analog signal $X_{3}(t)$, (d) waveform of analog signal $X(t)$, and (e) waveform of analog signal $Y(t)$.

As shown in Fig. 4(a), the SVD curve has a larger downward trend at the $\left[\begin{array}{ll}0 & 20\end{array}\right]$ interval, while it has a smaller downward trend at the [20 200] interval. Using the singular value cumulative difference algorithm, the maximum value of the singular value cumulative difference distribution curve can be obtained, which is the black dot on the curve in Fig. 4(b). The abscissa value of the black point is 25 , so the number of effective singular values was 25. The first 25 singular values were considered to be the corresponding singular values of the signal, while the smaller singular values in the latter part were the singular values corresponding to the noise.

The corresponding singular values of the noise information were set to zero, and then the SVD inverse operation was performed to reconstruct a new signal to obtain the denoised signal $S^{*}(t) . \quad S^{*}(t)$ and the trend term R obtained by ESMD above were reconstructed into the final 


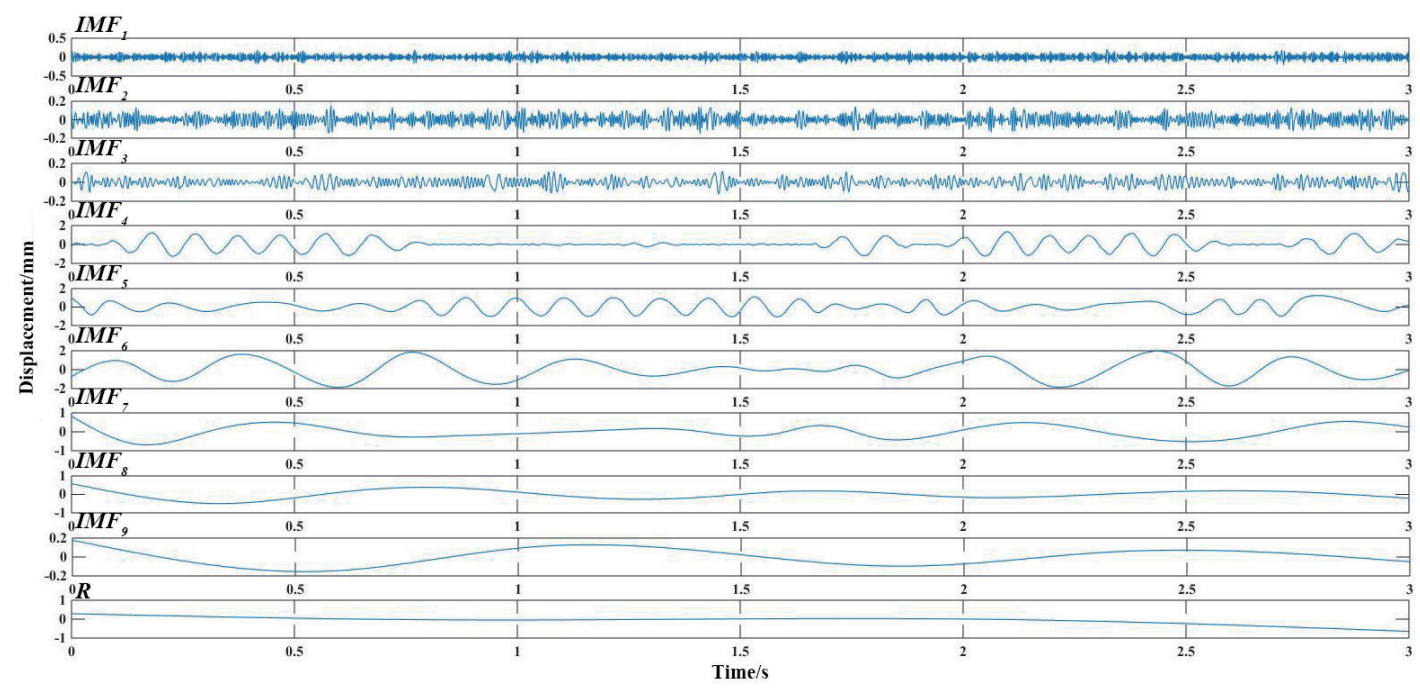

Fig. 3. (Color online) Decomposed IMFs of analog signal $Y(t)$ obtained by ESMD.

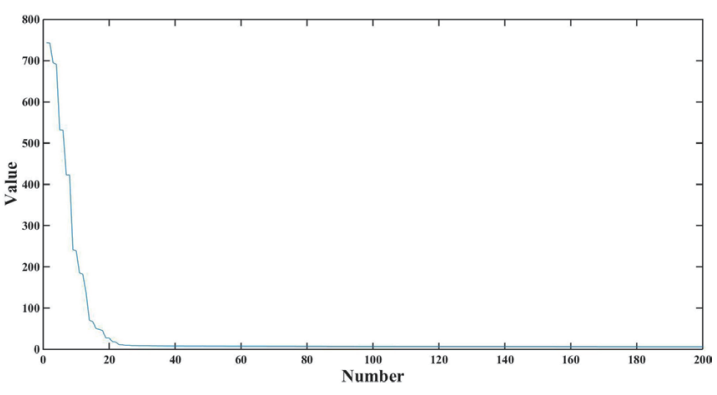

(a)

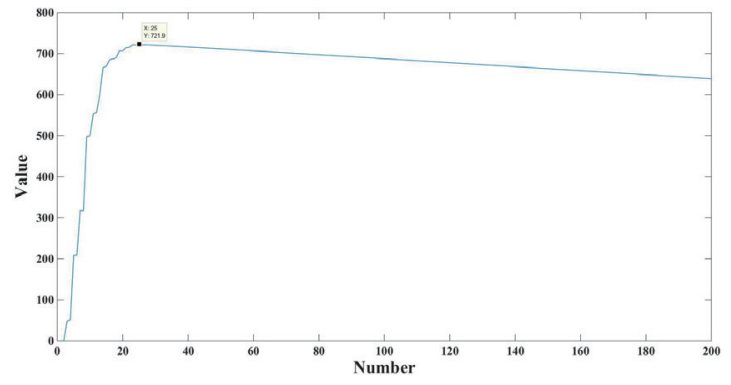

(b)

Fig. 4. (Color online) (a) SVD curve of reconstructed signal $S(t)$ and (b) singular value cumulative difference distribution curve of reconstructed signal $S(t)$.

denoised signal $Y^{*}(t)$. Figure 5(d) shows a comparison of the curves of the original analog signal $X(t)$ and the final denoised signal $Y^{*}(t)$. Moreover, to validate the accuracy and robustness of the proposed ESMD-SVD method for signal denoising, SVD, ESMD, and EEMD-SVD ${ }^{(29)}$ were selected for comparison with the proposed ESMD-SVD method. Figures 5(a)-5(c) show comparisons of the curves of the original analog signal $X(t)$ and the three denoised signals obtained by the three methods, respectively.

From Fig. 5, the following can be observed: (1) The waveforms of the denoised signals obtained by the four methods were generally consistent with that of the original analog signal $X(t)$, which indicated that the four denoising methods can retain the useful characteristics of signals. (2) The middle parts of the denoised signals obtained by the four denoising methods were basically the same as that of the original analog signal $X(t)$, which indicated that the four denoising methods can effectively eliminate the noise in the middle of the analog signal curve. (3) There were deviations from the original analog symbol in the peak parts of the curve for the denoised signals obtained by SVD and EEMD-SVD, as shown in Figs. 5(a) and 5(c), 


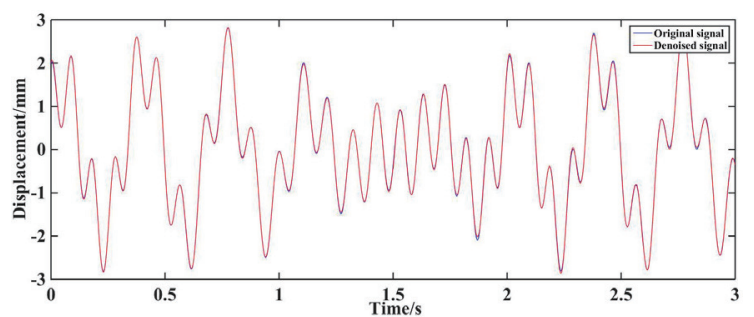

(a)

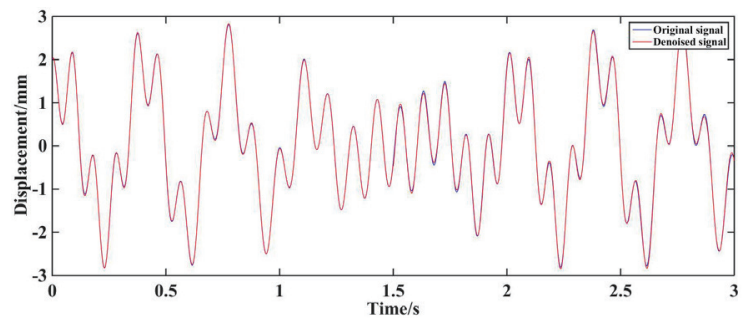

(c)

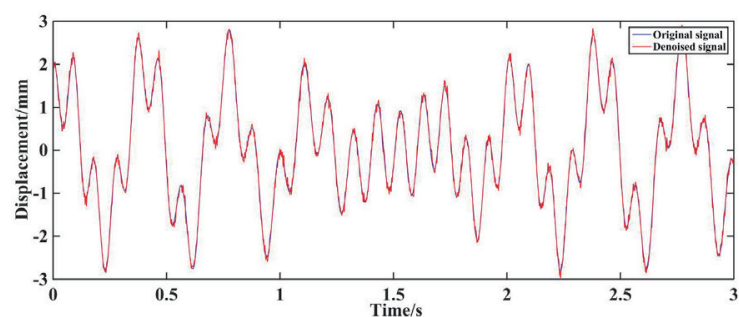

(b)

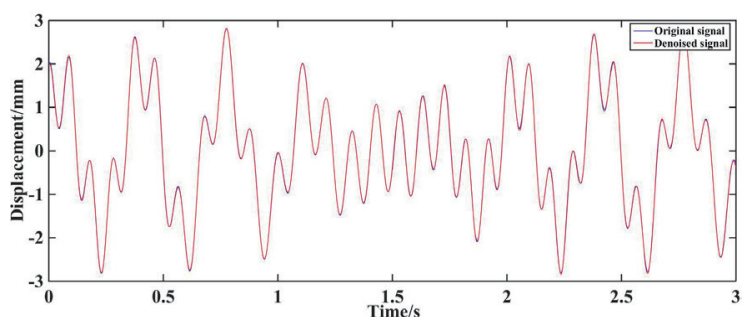

(d)

Fig. 5. (Color online) Comparisons of curves of signal $X(t)$ and denoised signals obtained by SVD, ESMD, EEMD-SVD, and ESMD-SVD. (a) $X(t)$ and signal denoised by SVD, (b) $X(t)$ and signal denoised by ESMD, (c) $X(t)$ and signal denoised by EEMD-SVD, and (d) $X(t)$ and $Y^{*}(t)$.

respectively. For the peak parts of the curves in Fig. 5(a), there was a maximum deviation of $0.008 \mathrm{~mm}$, and there was a maximum deviation of $0.006 \mathrm{~mm}$ for the peak parts of the curves in Fig. 5(c). As shown in Fig. 5(b), there was obvious noise in the peak part of the denoised signal curve obtained by ESMD. The results indicated that the proposed ESMD-SVD method not only has better denoising performance than the single SVD and single ESMD methods, but also has better denoising performance than EEMD-SVD, which combines SVD and EEMD.

In this study, two traditional objective evaluation indexes, SNR and root mean square error (RMSE), were introduced to evaluate the noise reduction effect. ${ }^{(14,15)}$ The evaluation indexes of the four noise reduction methods are shown in Table 1. The table reveals the following: (1) The two indexes of the denoised signal obtained by ESMD were the smallest among the four noise reduction methods, consistent with the results shown in Fig. 5. (2) The RMSE of the proposed ESMD-SVD denoising method was $0.0212 \mathrm{~mm}$, which was the smallest among the four denoising methods and close to zero. (3) The SNR of the analog signal $Y(t)$ was $20 \mathrm{~dB}$. The SNR of the signal denoised by SVD was $30.0317 \mathrm{~dB}$, which was $50.16 \%$ higher than that of the analog signal $Y(t)$. The SNR of the signal denoised by ESMD was $28.0857 \mathrm{~dB}$, which was $40.43 \%$ higher than that of the analog signal $Y(t)$. The SNR of the signal denoised by SVDEEMD was $33.3567 \mathrm{~dB}$, which was $66.78 \%$ higher than that of the analog signal $Y(t)$. The SNR of the signal denoised by ESMD-SVD was $35.8441 \mathrm{~dB}$, which was $79.22 \%$ higher than that of the analog signal $Y(t)$. The results show that compared with the other three denoising methods, the proposed ESMD-SVD method had a greater denoising ability for nonlinear and nonstationary signals. 
Table 1

SNR and RMSE values of signal denoised by four noise reduction methods.

\begin{tabular}{lcc}
\hline Index & SNR $(\mathrm{dB})$ & RMSE $(\mathrm{mm})$ \\
\hline SVD & 30.0317 & 0.0414 \\
ESMD & 28.0857 & 0.0517 \\
EEMD-SVD & 33.3567 & 0.0282 \\
ESMD-SVD & 35.8441 & 0.0212 \\
\hline
\end{tabular}

\section{On-site Experiment and Analysis}

In this study, in order to further verify the applicability and accuracy of the proposed ESMD-SVD denoising method to bridge signal denoising based on ground-based microwave interferometry, Fengbei Bridge was selected as the experimental bridge. Fengbei Bridge, as shown in Fig. 6(a), is one of the most important transportation hubs of West Fourth Ring Road in Beijing, China. In addition, there is a road intersection under the bridge. As shown in Fig. 6(b), we used an interferometric survey instrument IBIS-S, which is a typical system based on microwave interferometry. The instrument was located on one side of the bridge and no passive radar reflector was installed on the bridge. Owing to the surrounding environment, ground motion, complex traffic activities, manual operation, and the equipment itself, noise will inevitably exist in the collected bridge signals. IBIS-S consists of four parts: a radar sensor unit, a personal control computer, a power supply unit, and a tripod. The step frequency continuous wave (SFCW) and microwave interferometric techniques are the two main radar technologies of the radar senor unit. Under typical measurement conditions, the maximum sampling rate of the instrument was $200 \mathrm{~Hz}$, the maximum detection distance was $1 \mathrm{~km}$, the maximum distance resolution was $0.50 \mathrm{~m}$, and the maximum displacement measurement accuracy was $0.01 \mathrm{~mm}$. In this study, the angle of elevation of the radar sensor unit was set at $30^{\circ}$ so that the two antennas on the radar sensor unit could be aimed at the mid-span point of the central span of the bridge. The range resolution of the radar sensor was $0.5 \mathrm{~m}$, the sampling frequency was $200 \mathrm{~Hz}$, and the data acquisition time was $60 \mathrm{~s}$. Figure 7 shows the Fengbei Bridge signal obtained by IBIS-S with a sampling time of $15 \mathrm{~s}$.

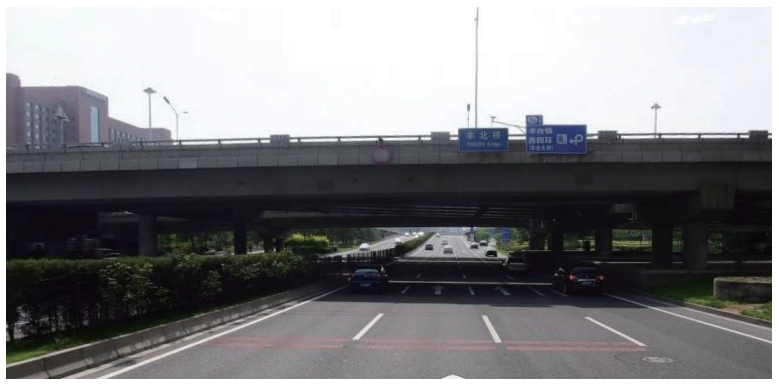

(a)

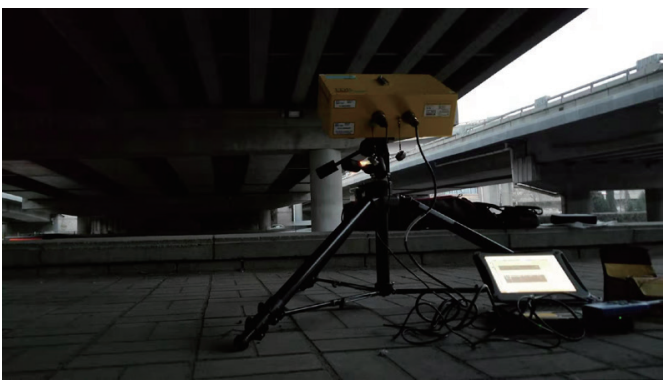

(b)

Fig. 6. (Color online) Fengbei Bridge and IBIS-S layout. (a) Fengbei Bridge and (b) dynamic deflection measurement of Fengbei Bridge using IBIS-S. 


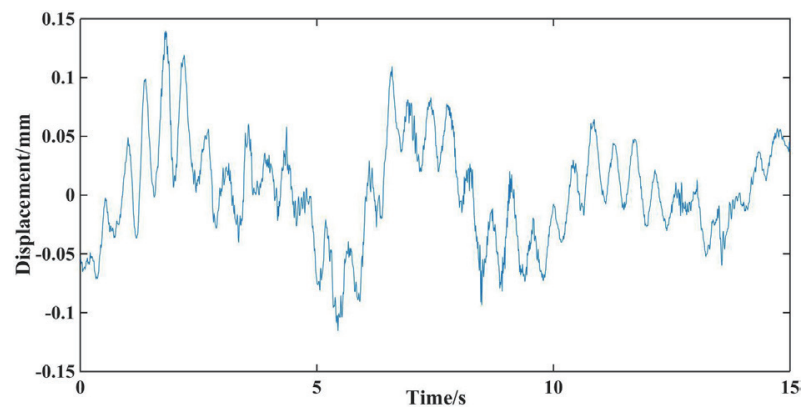

Fig. 7. (Color online) Waveform of signal of Fengbei Bridge obtained using IBIS-S.

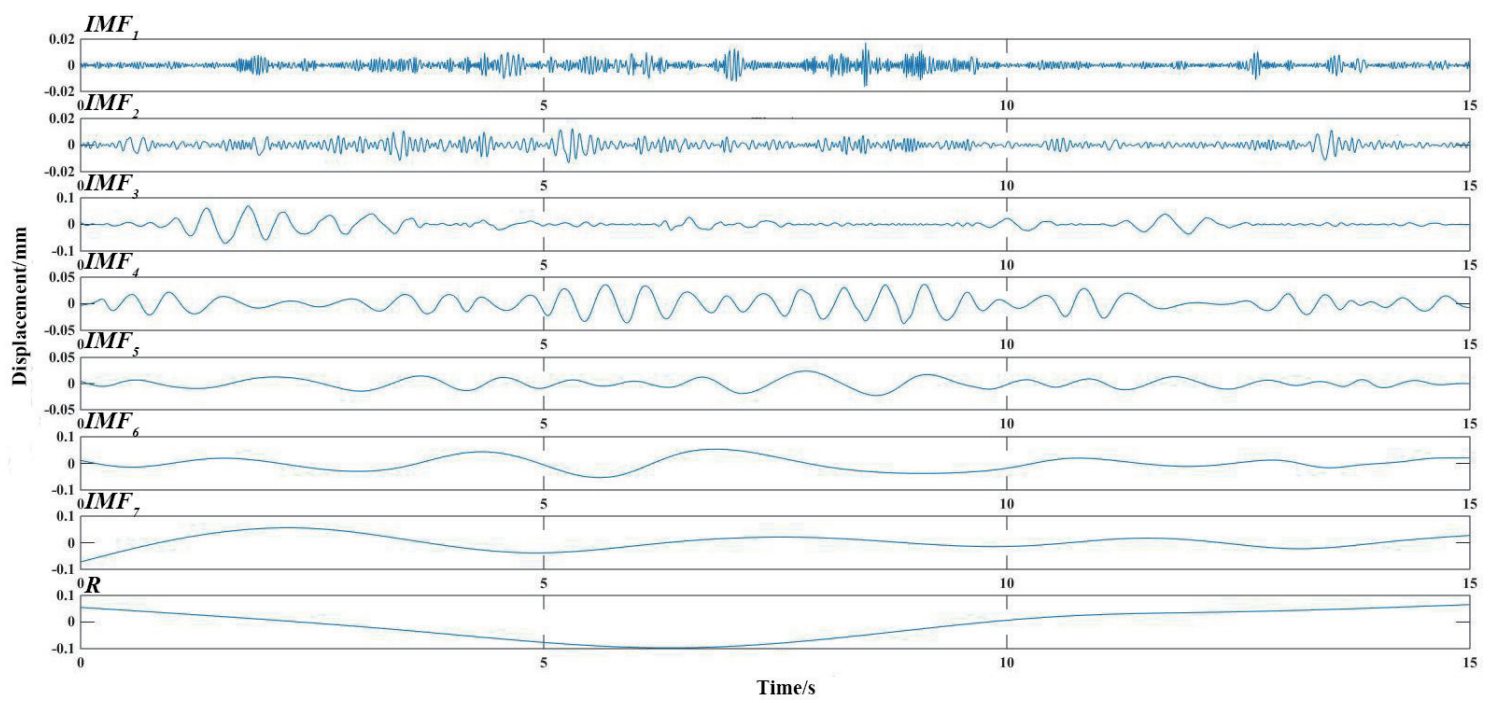

Fig. 8. (Color online) Decomposed IMFs of original bridge signal obtained by ESMD.

The original signal of Fengbei Bridge with IBIS-S was first decomposed into seven IMFs and one signal trend term R by ESMD, as shown in Fig. 8. Then, the SVD of the Hankel matrix was performed, and singular values were obtained. Since there are many singular values and the final values are small, only the first 200 singular values were selected to form the singular value distribution curve shown in Fig. 9(a). The singular value cumulative difference distribution curve was composed of the cumulative differences of the first 200 singular values as shown in Fig. 9(b). The maximum value of the singular value cumulative difference distribution curve was obtained using the singular value cumulative difference algorithm, which is the black dot on the curve in Fig. 9(b). The abscissa value of the black point was 46, so the number of effective singular values was 46 , and we determined that the first 46 singular values were the corresponding singular values of the signal. The final denoised signal (red curve) is shown in Fig. 10(d). Figures 10(a)-10(c) respectively show the results of comparing the original signal and the signals denoised by SVD, ESMD, and EEMD-SVD.

The inspection of the curves in Fig. 10 highlighted the following: (1) Compared with the single SVD denoising method, the waveform of the denoised signal obtained by ESMD-SVD 


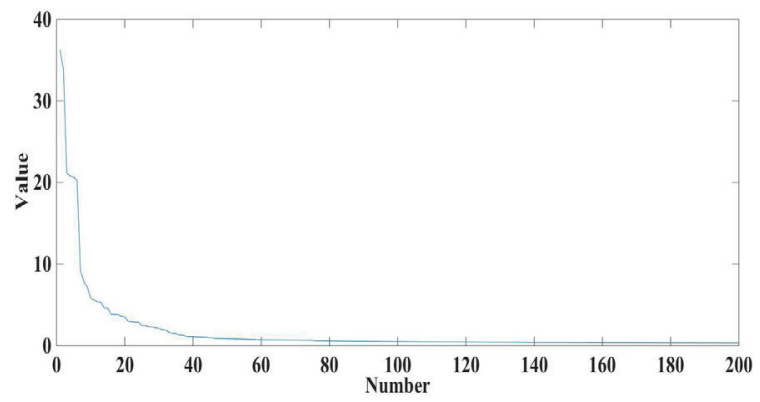

(a)

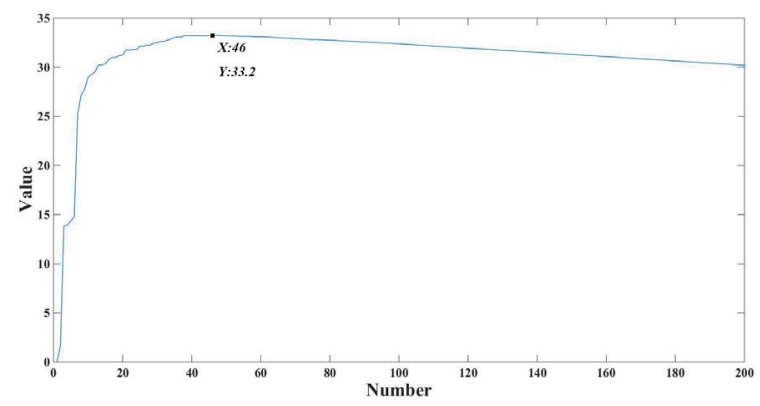

(b)

Fig. 9. (Color online) (a) Singular value distribution curve and (b) singular value cumulative difference distribution curve.

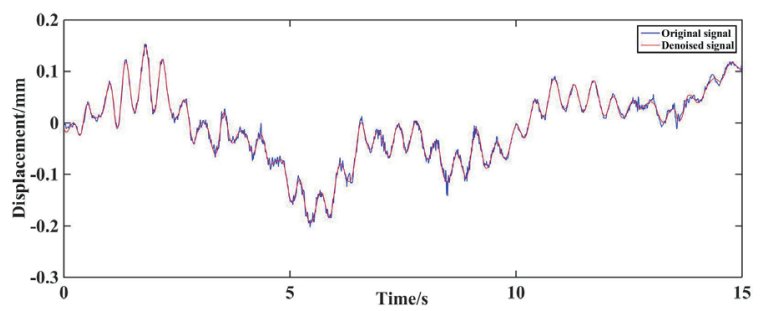

(a)

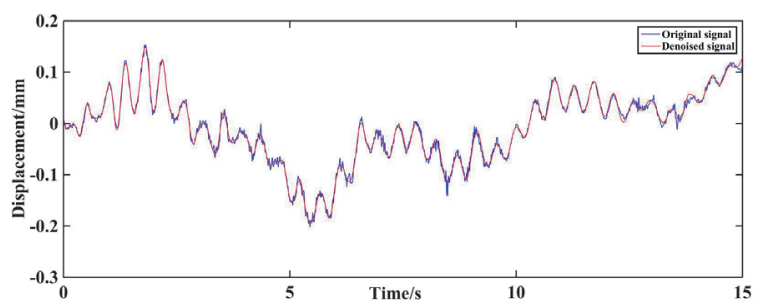

(c)

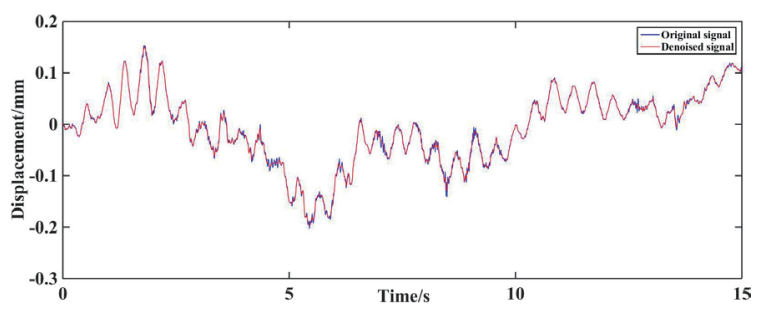

(b)

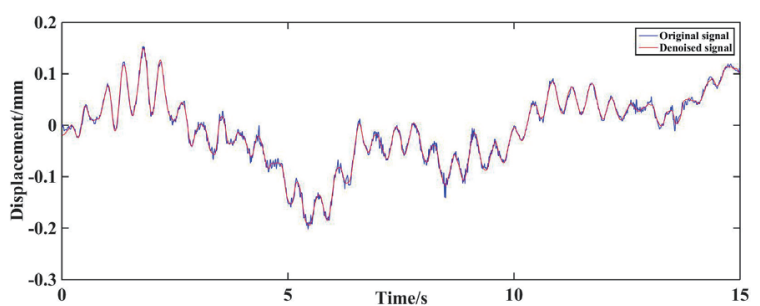

(d)

Fig. 10. (Color online) Comparison between denoised signals obtained by four denoising methods and original signal. Original and denoised signals obtained by (a) SVD, (b) ESMD, (c) SVD-EEMD, and (d) ESMD-SVD.

[red curve in Fig. 10(d)] was closer to that of the original signal. This shows that the proposed ESMD-SVD method can effectively eliminate the effect of the signal trend term on SVD and reduce the loss of useful details from the bridge signal. (2) Compared with the single ESMD noise reduction method, the denoised signal obtained by ESMD-SVD had smoother peaks and valleys. The result proves that the proposed method had a better noise reduction ability than ESMD for the peaks and troughs of the signal, and could effectively remove the random and impulse noises in the bridge signal obtained by ground-based microwave interferometry. (3) Between sampling times of 10 and $15 \mathrm{~s}$, owing to the effect of the mode mixing problem of EEMD, there was a large deviation between the denoised signal obtained by EEMD-SVD [red curve in Fig. 10(c)] and the original signal [blue curve in Fig. 10(c)]. Although ESMD effectively reduces the impact of the mode mixing problem, the problem still exists. Therefore, 
Table 2

Four objective evaluation indexes of denoised signals obtained by four denoising methods.

\begin{tabular}{lcccc}
\hline Index & NRR & SER & RVR & Z \\
\hline SVD & 3.9709 & 0.9972 & 0.2232 & 1 \\
ESMD & 7.1181 & 0.9990 & 0.3817 & 1.4585 \\
EEMD-SVD & 4.0280 & 0.9980 & 0.2234 & 1.4281 \\
ESMD-SVD & 10.8357 & 0.9980 & 0.2308 & 2.3731 \\
\hline
\end{tabular}

the first part of the denoised signal obtained by ESMD-SVD was also different from that of the original signal as shown in Fig. 10(d). Compared with that obtained by EEMD-SVD, the denoised signal obtained by ESMD-SVD was less affected by the mode mixing problem. In summary, the proposed ESMD-SVD method in the paper had a better noise reduction ability than the other methods of bridge signal denoising based on ground-based microwave interferometry.

In order to verify the noise reduction effect of ESMD-SVD, its signal denoising quality was evaluated by four objective evaluation indexes: noise suppression ratio (NRR), noise energy ratio (SER), root of variance ratio (RVR), and a composite index Z. ${ }^{14,15)}$ Table 2 shows the four evaluation indexes of the denoised signals obtained by SVD, ESMD, EEMD-SVD, and ESMD-SVD. The table highlights the following findings. (1) The NRR of 10.8357 for the proposed ESMD-SVD method was the largest among the four denoising methods. Therefore, ESMD-SVD had the strongest denoising ability. (2) The four denoising methods had similar SER and RVR values. In this case, a single index may lead to an incorrect judgment on the noise reduction quality, and it was necessary to adopt the comprehensive evaluation index $\mathrm{Z}$ to objectively evaluate the noise reduction quality of the four denoising methods. As shown in Table 2, Z was 2.3731 for ESMD-SVD, which was much better than the results for the other three denoising methods. The results prove that the ESMD-SVD denoising method proposed in this paper has good denoising ability, not only effectively reducing the effect of noise, but also retaining the characteristics of the signal.

\section{Conclusions}

In this study, in order to improve the accuracy of a bridge signal obtained by the IBIS-S sensor, an ESMD-SVD denoising method based on SVD and ESMD was proposed to remove the noise in the original bridge signal. Through simulated and real-data experiments, the following conclusions are drawn.

(1) The simulated experiment verified the feasibility and accuracy of the proposed ESMDSVD denoising method for nonlinear and nonstationary simulation signals. RMSE was $0.0212 \mathrm{~mm}$ and SNR was $35.8441 \mathrm{~dB}$ for ESMD-SVD, which were much better than the corresponding values for the other three denoising methods in this study. In addition, the SNR of ESMD-SVD was $79.22 \%$ higher than that of the analog signal $Y(t)$. The results show that ESMD-SVD can be applied to the denoising of nonlinear and nonstationary signals. 
(2) Compared with the single SVD method, ESMD-SVD can effectively reduce the effect of the signal trend term, and compared with ESMD, it can remove noise more effectively. Compared with EEMD-SVD, ESMD-SVD is less affected by the mode mixing problem. Therefore, the proposed denoising method can combine the advantages of SVD and ESMD, and its noise reduction ability is superior to those of SVD and ESMD.

(3) The real dynamic deflection signal of Fengbei Bridge was obtained using IBIS-S. The range resolution of the IBIS-S radar sensor was $0.5 \mathrm{~m}$ and the sampling frequency was $200 \mathrm{~Hz}$. The experimental results obtained with real data show that ESMD-SVD has a strong denoising ability, which not only effectively reduces the effect of noise on the bridge signal, but also retains the useful vibration characteristics of the bridge. In this paper, a comprehensive evaluation index $\mathrm{Z}$ is used to evaluate the quality of signal denoising, which avoids incorrect judgments in the evaluation of signal denoising quality. The comprehensive evaluation index $\mathrm{Z}$ of ESMD-SVD was 2.3731, which was much better than the results of the other three denoising methods.

\section{Acknowledgments}

This study was sponsored by the National Natural Science Foundation of China (grant no. 41871367), the Ministry of Science and Technology of the People's Republic of China (grant no. 2018YFE0206100), the Importation and Development of High-Caliber Talents Project of Beijing Municipal Institutions (grant no. CIT\&TCD201704053), the Science and Technology Project of Ministry of Housing and Urban-Rural Development of the People's Republic of China (grant no. 2017-K4-002), the Scientific Research Project of Beijing Educational Committee (grant no. KM201910016007), the Major Projects of Beijing Advanced Innovation Center for Future Urban Design (grant no. UDC2018031321), and the BUCEA Postgraduate Innovation Project.

\section{References}

1 X. Liu, S. Li, and X. Tong: IEEE J-STARS 11 (2018) 1. https://doi.org/10.1109/JSTARS.2018.2878482

2 T. A. Stabile, A. Perrone, M. R. Gallipoli, R. Ditommaso, and F. C. Ponzo: IEEE Geosci. Remote Sens. 10 (2013) 870. https://doi.org/10.1109/lgrs.2012.2226428

3 C. Negulescu, G. Luzi, M. Crosetto, and D. Raucoules: Eng. Struct. 51 (2013) 10. https://doi.org/10.1016/ j.engstruct.2013.01.005

4 X. Liu, Z. Lu, W. Yang, M. Huang, and X. Tong: Remote Sens. 10 (2018) 770. https://doi.org/10.3390/ rs10050770

5 M. Maizuar, L. Zhang, S. Miramini, P. Mendis, and R G. Thompson: Struct. Control Health Monit. 24 (2017) 1985. https:// doi.org/10.1002/stc.1985

6 L. Zhou, J. Guo, J. Hu, J. Ma, F. Wei, and X. Xue: Int. J. Remote Sens. 39 (2018) 1911. https://doi.org/10.1080/0 1431161.2017.1416698

7 L. Tian and B. Pan: Sensors 16 (2016) 134. https://doi.org/10.3390/s16091344

8 M. Pieraccini, M. Fratini, F. Parrini, and C. Atzeni: IEEE Trans. Geosci. Remote. 44 (2016) 3284. https://doi. org/10.1109/TGRS.2006.879112

9 H. Sun, Y. Zi, Z. He, J. Yuan, X. Wang, and L. Chen: Sensors 13 (2013) 1183. https://doi.org/10.3390/ s130101183

10 Z. Li, Z. He, Y. Zi, and Y. Wang: J. Sound Vib. 313 (2008) 342. https://doi.org/10.1016/j.jsv.2007.11.039

11 S. K. Yadav, R. Sinha, and P. K. Bora: IET Signal Process. 9 (2015) 88. https://doi.org/10.1049/ietspr.2014.0005 
12 R. Patil: Procedia Comput. Sci. 54 (2015) 849. https://doi.org/10.1016/j.procs.2015.06.099

13 C. Liu, C. Song, and Q. Lu: J. Appl. Geophys. 144 (2017) 125. https://doi.org/10.1016/j.jappgeo.2017.07.007

14 X. Liu, H. Wang, M. Huang, and W. Yang: Appl. Sci. 9 (2019) 3561. https://doi.org/10.3390/app9173561

15 X. Liu, M. Jiang, Z. Liu, and H. Wang: Shock Vib. 2020 (2020) 1. https://doi.org/10.1155/2020/8430986

16 N. E. Huang, Z. Shen, S. R. Long, M. C. Wu, and H. H. Liu: Proc. R. Soc. A 454 (1998) 903. https://doi. org/10.1098/rspa.1998.0193

17 J. B. Ali, N. Fnaiech, L. Saidi, B. Chebel-Morello, and F. Fnaiech: Appl. Acoust. 89 (2015) 16. https://doi. org/10.1016/j.apacoust.2014.08.016

18 X. Wei, R. Lin R, S. Liu, and C. Zhang: Shock Vib. 2016 (2016) 1. https://doi.org/10.1155/2016/7641027

19 A. R. Hassan and M. I. H. Bhuiyan: Comput. Meth. Prog. Bio. 140 (2017) 201. https://doi.org/10.1016/ j.cmpb.2016.12.015

20 A. Tabrizi, L. Garibaldi, A. Fasana, and S. Marchesiello: Meccanica 50 (2015) 865. https://doi.org/ 10.1007/ s11012-014-9968-z

21 X. Chen, G. Cheng, X. Shan, X. Hu, Q. Guo, and H. Liu: Measurement 73 (2015) 55. https://doi.org/10.1016/ j.measurement.2015.05.007

22 J. C. Chan, H. Ma, T. K. Saha, and C. Ekanayake: IEEE Trans. Dielect. Electr. Insul. 21 (2014) 294. https://doi. org/10.1109/tdei.2014.6740752

23 S. Wang, N. Zhang, L. Wu, and Y. Wang: Renew Energy 94 (2016) 629. https://doi.org/10.1016/ j.renene.2016.03.103

24 Z. Yang and J H. Zhong: Entropy 18 (2016) 112. https://doi.org/10.3390/e18040112

25 M. Zeng and Z. Chen: IEEE Trans. Ind. Electron. 1 (2019) 1. https://doi.org/10.1109/tie.2019.2898583

26 X. Yin, Y. Xu, X. Sheng, and Y. Shen: Sensors 19 (2019) 5032. https://doi.org/10.3390/s19225032

27 Z. Meng, Zi. Liu, and M. Lv: Chn. Mech. Eng. (2020) (in Chinese). http://kns.cnki.net/kcms/detail/42.1294. TH.20200630.1049.012.html

28 Y. Yang, A. Huo, and F. Qi: IOP Conf. Ser: Mater Sci Eng. 799 (2020) 012030. https://doi.org/10.1088/1757$899 x / 799 / 1 / 012030$

29 C. Wen and C. Zhou: Appl. Mech. Mater. 411-414 (2013) 1067. https://doi.org/10.4028/www.scientific.net/ amm.411-414.1067

30 Y. B. Yang, F. Xiong, Z. L. Wang, and H. Xu: Int. J. Struct. Stab. Dyn. 20 (2020) 04. https://doi.org/10.1142/ S0219455420500455

\section{About the Authors}

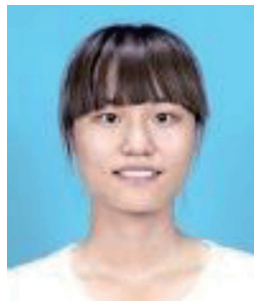

Mengzhuo Jiang received her B.S. degree in surveying and mapping engineering from Henan University of Engineering, China, in 2017. She is currently a graduate student majoring in geodesy and surveying engineering at Beijing University of Civil Engineering and Architecture. Her research interest is in the denoising of bridge dynamic deflection signals based on GBSAR.(2108160118005@stu.bucea.edu.cn)

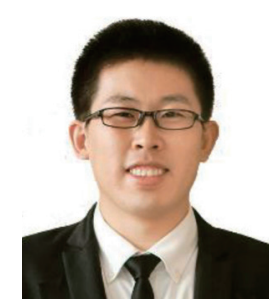

Xianglei Liu received his B.S. and M.S. degrees in geographic information systems from Shandong University of Science and Technology in 2005 and 2008, respectively. He received his Ph.D. degree in photogrammetry and remote sensing from Tongji University in 2012. He is currently a professor with Beijing University of Civil Engineering and Architecture. His research interests are in deformation monitoring based on GBSAR and high-speed videogrammetric measurement. 


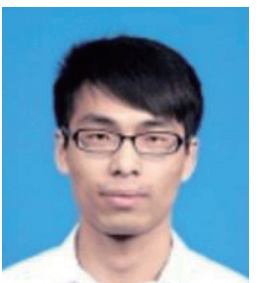

Hui Wang received his B.S. degree in remote sensing science and technology from Beijing University of Civil Engineering and Architecture, China, in 2018. He is currently a graduate student majoring in photogrammetry and remote sensing at Beijing University of Civil Engineering and Architecture. His research interest is in bridge dynamic deflection signal denoising based on GBSAR. (2108160218005@stu.bucea.edu.cn)

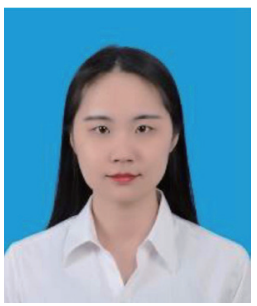

Yimeng Huang received her B.S. degree in remote sensing science and technology from Beijing University of Civil Engineering and Architecture, China, in 2020. She is currently a graduate student majoring in resources and environment at Beijing University of Civil Engineering and Architecture. Her research interest is in bridge dynamic deflection signal denoising based on GBSAR.(2108570020086@stu.bucea.edu.cn) 